\title{
Evolución de las características clínicas, ecográficas y patológicas de los pacientes con indicación de ecografía transrectal y biopsia de próstata entre 1994 Y 2003
}

\author{
F. Herranz Amo, J.Mª Díez Cordero, F. Verdú Tartajo, I. Castaño González, \\ M. Moralejo Gárate, J.I. Martínez Salamanca, R. Cabello Benavente, J.I. Monzó \\ Servicio de Urología. Hospital General Universitario “Gregorio Marañón”. Madrid.
}

Actas Urol Esp 2005; 29 (1): 55-63

\begin{abstract}
RESUMEN
EVOLUCIÓN DE LAS CARACTERÍSTICAS CLÍNICAS, ECOGRÁFICAS Y PATOLÓGICAS DE LOS PACIENTES CON INDICACIÓN DE ECOGRAFÍA TRANSRECTAL Y BIOPSIA DE PRÓSTATA ENTRE 1994 Y 2003

Objetivo: El objetivo de este artículo es evaluar los cambios en el tiempo que se han producido en las características clínicas, ecográficas, bioquímicas y patológicas de los pacientes sometidos a ecografia transrectal y biopsia de próstata en un periodo 10 años (1994-2003).

Material y método: Se ha analizado de forma retrospectiva los pacientes sometidos a una primera biopsia de próstata en el periodo 1994-2003. Durante el mismo se ha modificado en varias ocasiones los criterios de biopsia, por lo que el análisis de los resultados se realizará en 4 periodos de tiempo: 1994-1997, 1998, 1999-2003 (este último se desdobla en dos - 1999-2001 y 2002-2003 - para observar la evolución en el tiempo con los mismos criterios de biopsia). Los pacientes son asignados a grupos de riesgo según D’Amico.

Resultados: Hemos observado tanto en los sujetos remitidos para biopsia como en los diagnosticados de cáncer de próstata (CP) una disminución de la edad media ( $\mathrm{p}=0,0001$ y $\mathrm{p}=0,01)$, un aumento de los pacientes con PSA entre 4,1-10 ng/ml ( $\mathrm{p}=0,0001 \mathrm{y}$ $\mathrm{p}=0,0001)$, un aumento de los sujetos sin alteraciones al tacto rectal $(\mathrm{p}=0,0001$ y $\mathrm{p}=0,0001)$ ni ecográficas $(\mathrm{p}=0,0001 \mathrm{y} p=0,0001)$. La incidencia de diagnósticos de cáncer ha disminuido del 39,4\% al 34,3\% ( $\mathrm{p}=0,03$ ). Se observa un aumento del score 7 con disminución del 5-6, 8-10 y desaparición del 2-4 (p=0,0001). Los pacientes asignados al grupo de bajo riesgo aumentan del 9\% al 18,1\%, del $20,2 \%$ al $43,1 \%$ los asignados al grupo de riesgo intermedio y disminuyen los asignados al grupo de riesgo alto del $70,7 \%$ al $38,7 \%(\mathrm{p}=0,0001)$. Al analizar los dos últimos periodos de tiempo se mantiene la evolución de la serie excepto en la disminución de la edad de biopsia y de diagnostico de cáncer.

Conclusiones: Los pacientes con indicación de biopsia tienen actualmente unos factores de riesgo con menor peso que hace una década. Actualmente diagnosticamos a los pacientes de CP con unos factores pronósticos más favorables. Pero el precio que tenemos que pagar por esta anticipación diagnóstica se traduce en: una menor efectividad de la biopsia, un aumento de la población sin CP sometida a las complicaciones físicas y al estrés psicológico que implica la realización de la biopsia, un aumento de pacientes sometidos a re-biopsia y por lo tanto, un incremento en la utilización de los recursos y de los costes necesarios para el diagnostico de CP. Palabras claves: Ecografia transrectal. Biopsia de próstata ecodirigida. Cáncer de próstata. Evolución en el tiempo.
\end{abstract}

\section{ABSTRACT}

EVOLUTION OF THE CLINICAL, ECHOGRAPHIC AND PATHOLOGICAL CHARACTERISTICS OF PATIENTS WITH INDICATION OF TRANSRECTAL ULTRASOUND AND PROSTATE BIOPSY BETWEEN 1994 AND 2003

Objective: The aim of this paper is to evaluate the evolution in the clinical, echographic and pathological characteristics of patients undergoing transrectal ultrasound and prostate biopsy over a 10 year period (1994-2003).

Material and methods: Patients undergoing a first biopsy of the prostate between 1994-2003 were studied retrospectively. Since the biopsy criteria have changed several times over this period, results were analysed for four different periods: 1994-1997, 1998, 1999-2003 (the latter was further divided into two periods - 1999-2001 and 2002-2003 - to observe the temporal evolution with the same biopsy criteria). Patients were assigned to risk groups according to D`Amico.

Results: We observed that individuals referred for biopsy and those diagnosed with prostate cancer (PC) had a lower mean age $(\mathrm{p}=0.0001$ and $\mathrm{p}=0.01)$, there were more patients with a PSA from $4.1-10 \mathrm{ng} / \mathrm{ml}(\mathrm{p}=0.0001$ and $\mathrm{p}=0.0001)$, more patients had no significant DRE findings $(\mathrm{p}=0.0001$ and $\mathrm{p}=0.0001)$ or ultrasound findings $(\mathrm{p}=0.0001$ and $\mathrm{p}=0.0001)$. The incidence of cancer diagnosis has decreased from $39.4 \%$ to $34.3 \%(\mathrm{p}=0,03)$. There was an increased incidence of score 7 , at the expense of a decline in scores 5-6 and 8-10 and disappearance of score $2-4(\mathrm{p}=0.0001)$. Patients assigned to the low risk group increased from $9 \%$ to $18.1 \%$, those assigned to the intermediate risk group from $20.2 \%$ to $43.1 \%$ and there was a reduction in those assigned to the high risk group from $70.7 \%$ to $38.7 \%$ ( $\mathrm{p}=0.0001$ ). Analysis of the final two time periods revealed that the evolution of the series remained the same except for a decrease in age at biopsy and diagnosis of cancer.

Conclusions: Nowadays, the risk factors of patients with an indication of biopsy have less weight than ten years ago. We currently diagnose patients with PC with more favourable prognostic factors. However, the price we pay for this earlier diagnosis is reflected in a less effective biopsy, a larger proportion of the population without PC having to experience the physical complications and psychological stress of a biopsy, a greater number of patients having to undergo a second biopsy and, therefore, a greater and more costly use of resources to diagnose PC.

Key words: Transrectal ultrasound. Echo-guided prostate biopsy. Prostate cancer. Evolution over time. 
$\mathrm{E}^{1}$ 1 cáncer de próstata es la neoplasia no cutánea más frecuentemente diagnosticada en USA y la segunda causa de muerte por enfermedad oncológica después del cáncer de pulmón. Se estima que en el año 2004 se diagnosticaran en USA 230.110 nuevos casos y morirán por esta enfermedad 29.900 hombres $^{1}$. La incidencia en la Comunidad de Madrid en el año 2000 fue de 100,4 casos x 100.000 hombres $^{2}$.

La American Cancer Society ${ }^{3}$ y la American Urological Association ${ }^{4}$ recomiendan la realización anual de un tacto rectal y una determinación de PSA en los sujetos mayores de 50 años y una expectativa de vida superior a los 10 años, siendo deseable que se aporte información sobre los riesgos y posibles beneficios del diagnostico precoz del cáncer de próstata. La introducción del PSA en la clínica y la biopsia transrectal ecodirigida han producido un aumento de los pacientes sometidos a biopsia ${ }^{5}$ con una efectividad que oscila entre el $11 \%$ y el $44 \%^{6,7}$, de los diagnósticos de cáncer ${ }^{8}$, así como una migración en el estadio y en los niveles de PSA ${ }^{9}$.

El objetivo de este artículo es evaluar los cambios en el tiempo que se han producido en las características clínicas, ecográficas, bioquímicas y patológicas de los pacientes sometidos a ecografia transrectal y biopsia de próstata en un periodo 10 años (1994-2003).

\section{MATERIAL Y MÉTODO}

La ecografia transrectal (ETR) y biopsia de próstata ecodirigida se introdujeron en nuestro Centro a finales de 1993. Desde 1994 se recogieron de forma prospectiva en una base de datos de SPSS distintas variables clínicas, ecográficas y patológicas de las biopsias realizadas por el autor. Los criterios para la indicación de biopsia han evolucionado con el paso del tiempo: 1- Desde 1994 a 1997 se indicó biopsia a todos los pacientes con tacto rectal sospechoso o PSA mayor de $10 \mathrm{ng} / \mathrm{ml}$, cuando el tacto rectal era normal y el PSA estaba entre $4-10 \mathrm{ng} / \mathrm{ml}$. solo se biopsiaron los pacientes en los que se identificó un nódulo ecográfico de distinta ecogenicidad o los que presentaron un PSAD > 0,15; 2 - En 1998 se cambio la técnica de determinación de PSA en nuestro Centro y se realizó un estudio para definir el punto de corte efectivo del PSA y del índice de PSA ${ }^{10}$. Se indicó biopsia a todos los pacientes con tacto rectal sospechoso o PSA > 2,5 ng/ml.; 3 - Desde 1999 hasta el 2003 se indicó biopsia de próstata a todos los pacientes con tacto rectal sospechoso o PSA > $10 \mathrm{ng} / \mathrm{ml}$., en los pacientes con tacto rectal normal y PSA entre 3,5 y $10 \mathrm{ng} / \mathrm{ml}$. se efectuó biopsia cuando el índice de PSA (PSA libre dividido por PSA total) fue inferior a 27.

Todos los pacientes realizaron preparación rectal mediante enemas de limpieza y profilaxis antibiótica oral. La ecografia transrectal se realizó con un ecógrafo Brüel \& Kjaer modelo 3535 con un transductor transrectal multiplanar a 7,5 Mhz. con canal de biopsia integrado tipo 8551 , colocándose al paciente en posición de litotomía. El 97\% de las exploraciones fueron realizadas por el autor y el resto por un MIR supervisado por el autor.

La exploración se inició con la realización de un tacto rectal clasificándose los pacientes según la TNM actual en ese momento, para el estudio se han reclasificado en la base de datos todos los pacientes a la TNM de 1997. Se consideró como tacto rectal sospechoso cuando se identificó un nódulo o una zona de mayor consistencia que el resto de la glándula, las próstatas con una mayor consistencia de forma global no se consideraron como sospechosas.

Tras la introducción del transductor se calculó el volumen de la glándula prostática según la formula del elipsoide (cálculo realizado por el software incluido en el ecógrafo). Se consideró como nódulo ecográfico identificable, toda alteración de la ecogenicidad en la zona periférica. Se realizó biopsia transrectal de la glándula prostática con un sistema automático de punción y agujas calibre $18 \mathrm{G}$ que extraen cilindros de $17 \mathrm{~mm}$. de longitud.

Las biopsias se realizaron en la parte más lateral de cada lóbulo prostático intentando incluir en ellas la mayor cantidad de tejido de la zona periférica y los nódulos de distinta ecogenicidad que se identificaron. A los pacientes con tacto rectal no sospechoso y a los que tenían un nódulo palpable susceptibles de prostatectomía se les realizaron 3 biopsia de cada lóbulo, a los pacientes con nódulo palpable y no susceptibles de prostatectomía se les realizaron entre 2 y 4 biopsias. La media de punciones de la serie fue de $5 \pm 1,2(2-8)$. 
De la base de datos se han extraídos los casos sometidos a la primera biopsia con el objetivo de evaluar si se han producido modificaciones en las características clínicas, ecográficas y patológicas. Debido a los cambios en las indicaciones de biopsia ya comentados podemos identificar 3 periodos bien diferenciados - 1994-1997, 1998 y 1999-2003 - en los que las indicaciones de biopsia fueron distintas.

Comparamos la evolución anual de las siguientes variables: edad, PSA, hallazgos al tacto rectal, hallazgos en la ETR, existencia de cáncer en la biopsia y score de Gleason. Para su análisis estadístico hemos estratificado el periodo de 10 años de la serie en 4 grupos: 1994-1997, 1998, 1999-2000 y 2001-2003.

Hemos dividido el periodo 1999-2003 en dos con el objetivo de detectar si existen variaciones en el tiempo aún con las mismas indicaciones de biopsia. Los pacientes con cáncer de próstata fueron agrupados en grupos de riesgo según los criterios de $\mathrm{D}^{-}$Amico $^{11}$ con el ajuste a la clasificación TNM-97 realizada por Cooperberg ${ }^{12}$ :

1. Riesgo bajo: PSA $\leq 10 \mathrm{ng} / \mathrm{ml}$., score $\leq 6 \mathrm{y}$ estadio T1-2a;

2. Riesgo intermedio: PSA entre 10,1 y 20 ng/ml. o score 7 o estadio T2b;

3. Riesgo elevado: $\mathrm{PSA}>20 \mathrm{ng} / \mathrm{ml}$. o score $\geq 8$ o estadio T3-4. Para las variables cualitativas se aplicó el test de la Chi cuadrado, ANOVA para el resto de las variables cuantitativas, excepto el PSA que se utilizó el test de Kruskal-Wallis.

Para la representación gráfica de los resultados hemos utilizados gráficos de porcentaje acumulativo por años.

\section{RESULTADOS}

En el periodo comprendido entre 1994 y 2003 se realizaron 4.133 ETR y biopsia de próstata, de ellas $3.284(79,5 \%)$ fueron primeras biopsia y van a ser la población diana de este estudio. Se excluyeron 206 (6,3\%) pacientes por pérdida de los siguientes datos: edad - 35 (1\%), PSA - 90 (2,7\%), tacto rectal - $21(0,6 \%)$, volumen ecográfico - 27 $(0,8 \%)$, nódulo ecográfico - $1(0,03 \%)$ y anatomía patológica - 32 (1\%). Por lo tanto, quedan 3078 $(93,7 \%)$ pacientes válidos para el estudio.

La edad media de la serie fue de 68,2 $\pm 7,4$ (37-92) con una mediana de 69 años. La media de PSA fue de 43,9 \pm 556,3 (0,1 - 28600) con una mediana de $10 \mathrm{ng} / \mathrm{ml}$. En la Tabla 1 se describen las características clínicas de los paciente sometidos a ETR-biopsia y de los pacientes con diagnostico de cáncer de próstata. En la Tabla 2 se relacionan los hallazgos al tacto rectal con los ecográficos. Los pacientes con tacto rectal no sospechoso y sin nódulo ecográfico identificable representaron el 52,3\% (1610) de la serie. En el $37,8 \%$ (1164) de los pacientes se confirmó la existencia de un cáncer de próstata. La edad media de los pacientes con cáncer fue de $70 \pm 7,4$ años frente a los $67 \pm 7,3(\mathrm{p}=0,0001)$ de los sujetos $\sin$ cáncer. Los pacientes con cáncer presentaron una media de PSA de 88,5 $\pm 888,6 \mathrm{ng} / \mathrm{ml}$. frente a 16,8 $\pm 125,5(\mathrm{p}=0,001)$ de los casos sin cáncer.

La relación entre los hallazgos al tacto rectal y los ecográficos en los pacientes con cáncer se detalla en la Tabla 3.

\section{Tabla 1}

Características clínicas de los pacientes sometidos a una primera ecografía transrectal y biopsia de próstata.

\begin{tabular}{|c|c|c|}
\hline & $\begin{array}{c}\text { Total serie } \\
(3.078) \\
\text { n (\%) }\end{array}$ & $\begin{array}{l}\text { Pacientes con } \\
\text { cáncer } \\
(1.164) \\
\text { n }(\%) \\
\end{array}$ \\
\hline $\begin{array}{l}\text { Edad (años): } \\
\quad<40 \\
41-50 \\
51-60 \\
61-70 \\
71-80 \\
>80\end{array}$ & $\begin{array}{c}2(0,06) \\
35(1,1) \\
442(14,4) \\
1371(44,6) \\
1110(36,1) \\
118(3,8)\end{array}$ & $\begin{array}{c}0 \\
10(0,8) \\
116(10) \\
457(39,3) \\
507(43,6) \\
74(6,4)\end{array}$ \\
\hline $\begin{array}{l}\text { PSA (ng/ml.): } \\
\leq 4 \\
4,1-10 \\
10,1-20 \\
>20\end{array}$ & $\begin{array}{l}139(4,5) \\
1417(46) \\
913(29,7) \\
609(19,8)\end{array}$ & $\begin{array}{c}34(2,9) \\
418(35,9) \\
320(27,5) \\
392(33,7)\end{array}$ \\
\hline $\begin{array}{l}\text { Tacto rectal (TNM-97): } \\
\text { T0 } \\
\text { T2a } \\
\text { T2b } \\
\text { T3-4 }\end{array}$ & $\begin{array}{l}2049(66,6) \\
556(18,1) \\
209(6,8) \\
264(8,6)\end{array}$ & $\begin{array}{l}387(33,2) \\
366(31,4) \\
168(14,4) \\
243(20,9)\end{array}$ \\
\hline $\begin{array}{l}\text { Volumen próstata (cc.): } \\
\text { Nódulo ecográfico: } \\
\text { SI } \\
\text { NO }\end{array}$ & $\begin{array}{l}58,1 \pm 34,1 \\
1336(43,4) \\
1742(56,6)\end{array}$ & $\begin{array}{l}45,9 \pm 27,1 \\
828(71,1) \\
336(28,9)\end{array}$ \\
\hline $\begin{array}{l}\text { Score Gleason: } \\
\text { No valorable } \\
\begin{array}{l}2-4 \\
5-6 \\
7 \\
8-10\end{array}\end{array}$ & & $\begin{array}{c}18(1,5) \\
62(5,3) \\
284(24,4) \\
347(29,8) \\
453(38,9)\end{array}$ \\
\hline $\begin{array}{l}\text { Grupo de riesgo: } \\
\text { Desconocido } \\
\text { Bajo } \\
\text { Intermedio } \\
\text { Alto }\end{array}$ & & $\begin{array}{c}18(1,5) \\
173(14,9) \\
335(28,8) \\
638(54,8)\end{array}$ \\
\hline
\end{tabular}


Tabla 2

Relación entre los hallazgos del tacto rectal y la ecografía en los pacientes sometidos a una primera biopsia de próstata.

\begin{tabular}{lccccc}
\hline Nódulo & \multicolumn{5}{c}{ Hallazgos al tacto rectal } \\
\cline { 2 - 6 } ecográfico & $\mathbf{T 0}$ & $\mathbf{T 2 a}$ & $\mathbf{T 2 b}$ & $\mathbf{T 3 a}$ & $\mathbf{T 4}$ \\
& $\mathbf{n}(\%)$ & $\mathbf{n ~ ( \% )}$ & $\mathbf{n}(\%)$ & $\mathbf{n}(\%)$ & $\mathbf{n ~ ( \% ) ~}$ \\
\hline SI & $439(21,4)$ & $467(84)$ & $178(85,2)$ & $232(95,5)$ & $20(95,2)$ \\
NO & $1610(78,6)$ & $89(16)$ & $31(14,8)$ & $11(4,5)$ & $1(4,8)$ \\
\hline
\end{tabular}

Tabla 3

Relación entre los hallazgos del tacto rectal y la ecografía en los pacientes diagnosticados de cáncer de próstata en la primera biopsia de próstata.

\begin{tabular}{lccccc}
\hline Nódulo & \multicolumn{5}{c}{ Hallazgos al tacto rectal } \\
\cline { 2 - 6 } ecográfico & To & T2a & T2b & T3a & T4 \\
& $\mathbf{n}(\%)$ & $\mathbf{n}(\%)$ & $\mathbf{n}(\%)$ & $\mathbf{n}(\%)$ & $\mathbf{n}(\%)$ \\
\hline SI & $111(28,7)$ & $331(90,4)$ & $154(91,7)$ & $214(95,5)$ & $18(95)$ \\
NO & $276(71,3)$ & $35(9,6)$ & $14(8,3)$ & $10(4,5)$ & $1(5)$ \\
\hline
\end{tabular}

nuyó desde un $82 \%$ hasta un $56,6 \%(\mathrm{p}=0,0001)$.

Incidencia de cáncer y score de Gleason (Fig. 4) - La incidencia de $\mathrm{CP}$ ha disminuido desde un $39,4 \%$ hasta un $34,3 \% \quad(p=0,03)$. Se observa un aumento progresivo del score 7 , una disminución de los score 8-10 desde el año 1998 y una desaparición de los score 2-4 desde el año 2000 ( $\mathrm{p}=0,0001)$.

Grupos de riesgo (Fig. 5) - Se observa un aumento progresivo del grupo de bajo riesgo desde un $9 \%$ hasta un $18,1 \%$,
Los pacientes diagnosticados de cáncer con tacto rectal no sospechoso y sin nódulo ecográfico identificable representaron el 23,7\% (276) del total de los casos.

Comparación entre los distintos periodos:

Edad media - La edad media en los pacientes remitidos para ETR y biopsia ha disminuido en los distintos periodos $(69,4 \pm 7,3 ; 67,9 \pm 7,6 ; 67,5$ $\pm 7,5$ y $67,5 \pm 7,2 ; \mathrm{p}=0,0001)$. En los pacientes diagnosticados de $\mathrm{CP}$ también se ha observado esta disminución de la edad al diagnostico $(70,7$ $\pm 7,4 ; 70,3 \pm 7,2 ; 69,3 \pm 7,4$ y 69,1 $\pm 7,4 ; p=0,01)$.

Antígeno específico prostático (Fig. 1) - Los pacientes que se remitieron a ETR y biopsia con PSA entre 4,1-10 ng/ml. han aumentado de forma muy importante, desde un $29,3 \%$ hasta un $64,7 \%$ ( $\mathrm{p}=0,0001)$. Esto se ha mantenido en el subgrupo de pacientes diagnosticados de cáncer de próstata, de $25,1 \%$ a $54,1 \%$ ( $\mathrm{p}=0,0001)$.

Tacto rectal (Fig. 2) - El porcentaje de pacientes con tacto rectal no sospechoso que fueron remitidos para ETR y biopsia aumentó desde un $57,9 \%$ hasta un 76,7\% (p=0,0001). En los pacientes diagnosticados de $\mathrm{CP}$ se ha mantenido este aumento de $23,4 \%$ a $45,9 \%$ ( $\mathrm{p}=0,0001)$.

Nódulo ecográfico (Fig. 3) - En los pacientes sometidos a ETR y biopsia el porcentaje en los que se identificó un nódulo ecográfico disminuyo desde el 60,1\% hasta el 30,2\% (p=0,0001). De la misma forma en los sujetos diagnosticados de $\mathrm{CP}$ la identificación de un nódulo ecográfico dismi- al igual que un aumento del grupo de riesgo intermedio de $20,2 \%$ a $43,1 \%$ y una disminución del grupo de alto riesgo de $70,7 \%$ a $38,7 \%$ $(\mathrm{p}=0,0001)$.

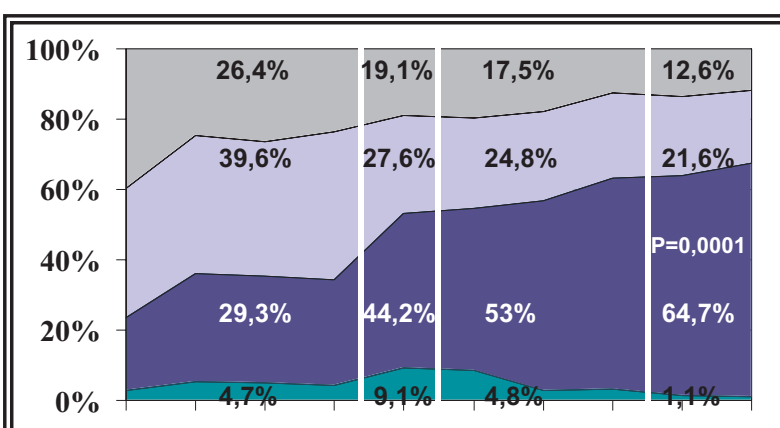

1994199519961997199819992000200120022003

$$
<4 \square 4,1 \text { - } 10 \square 10,1 \text { - } 20 \square>20
$$

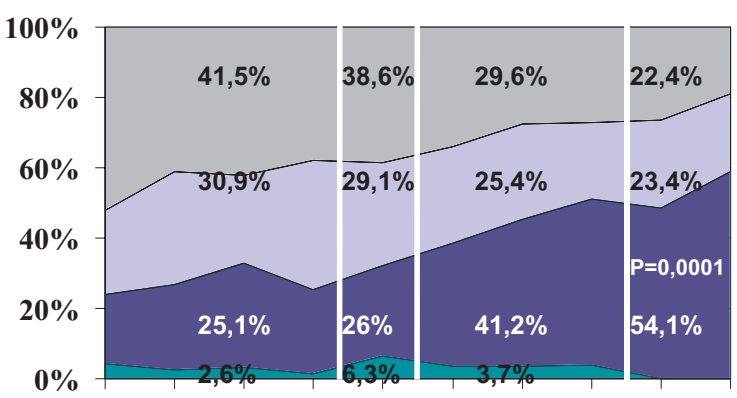

1994199519961997199819992000200120022003

$$
<4 \square 4,1 \text { - } 10 \square 10,1 \text { - } 20 \square>20
$$

FIGURA I. Evolución del PSA en los pacientes sometidos a ETR-biopsia (gráfico superior) y en los pacientes con diagnóstico de cáncer de próstata (gráfico inferior). 


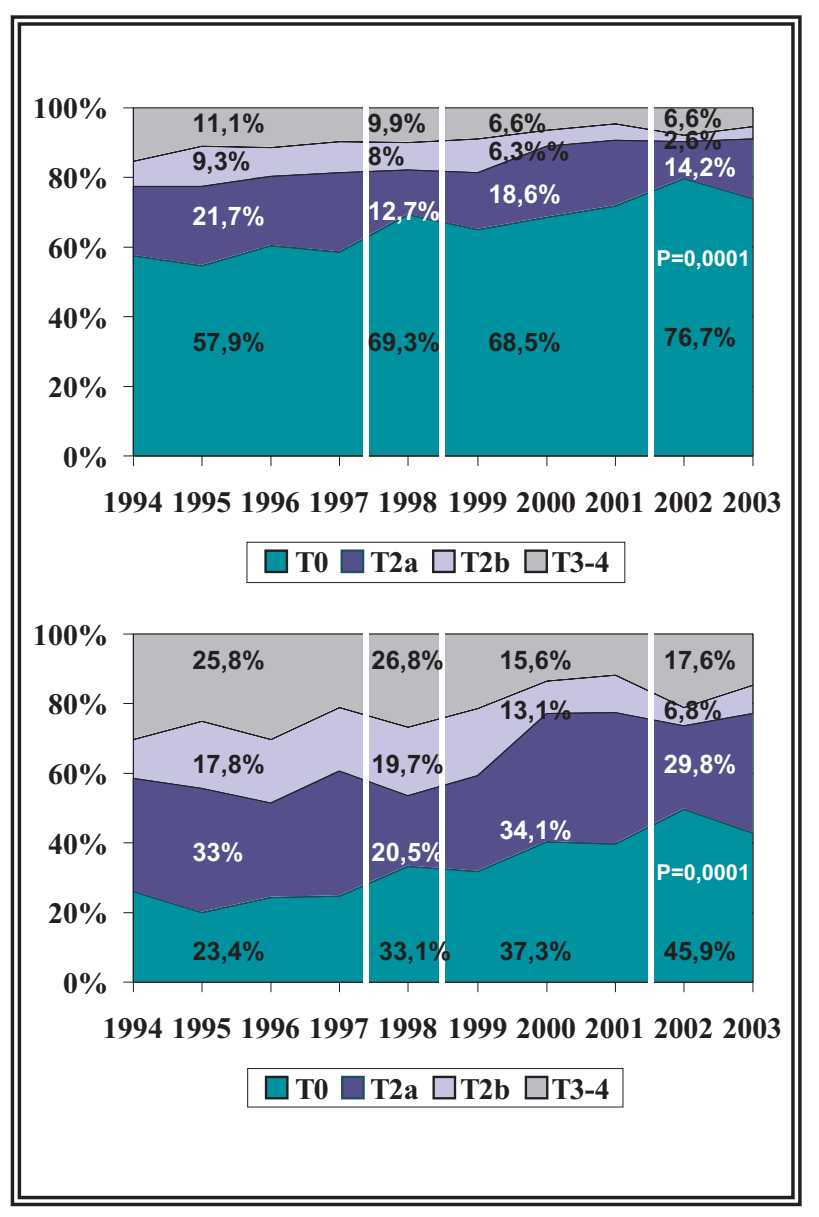

FIGURA 2. Evolución de los hallazgos al tacto rectal en los pacientes sometidos a ETR-biopsia (gráfico superior) y en los pacientes con diagnóstico de cáncer de próstata (gráfico inferior).

En el grupo de bajo riesgo se observa un aumento importante de los pacientes sin alteraciones al tacto rectal (Fig. 6) desde un 47,4\% hasta un $81,1 \%(\mathrm{p}=0,007)$ y una disminución hasta la desaparición de los scores inferiores a 5 $(\mathrm{p}=0,0001)$.

En el grupo de riesgo intermedio (Fig. 7) se observa un aumento de los pacientes con PSA < $10 \mathrm{ng} / \mathrm{ml}$. (de $25,9 \%$ a $67 \%, \mathrm{p}=0,0001$ ), una disminución de los pacientes en estadio T2b (de $17,3 \%$ a $3,4 \%, \mathrm{p}=0,01$ ) y un aumento del score 7 (de $50,6 \%$ a $92 \%, \mathrm{P}=0,0001$ ).

En el grupo de riesgo elevado (Fig. 8) se mantienen sin cambios los niveles de PSA $(\mathrm{p}=0,4)$ y los hallazgos al tacto rectal $(\mathrm{p}=0,4)$, mientras que se detecta una disminución de los score 8-10 (de $72,1 \%$ a $64,6 \%, \mathrm{P}=0,0001)$.
Comparación entre el periodo 1999-2000 y el 2001-2003:

No se han detectado cambios en la edad de los pacientes remitidos para ETR y biopsia $(\mathrm{p}=0,8) \mathrm{ni}$ en los diagnosticados de CP $(p=0,7)$. Los pacientes con PSA entre 4,1-10 ng/ml. han aumentado tanto en los sujetos remitidos a ETR y biopsia $(\mathrm{P}=0,0001)$ como en los diagnosticados de $C P(p=0,002)$, al igual que los pacientes sin anormalidades en el tacto rectal $(\mathrm{p}=0,0001$ y $\mathrm{p}=0,04)$, disminuyendo los pacientes con nódulos ecográficos $(p=0,04$ y $p=0,02)$. La incidencia de $\mathrm{CP}$ en las biopsias ha disminuido $(\mathrm{p}=0,0001)$, ha aumentado la proporción de score $7(\mathrm{p}=0,004)$ y existe una tendencia a aumentar los pacientes clasificados en el grupo de riesgo intermedio a costa fundamentalmente del grupo de alto riesgo $(\mathrm{p}=0,09)$.

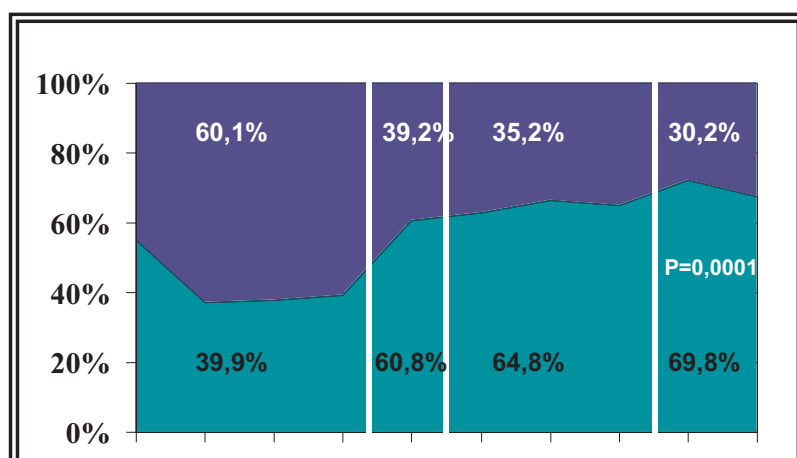

1994199519961997199819992000200120022003

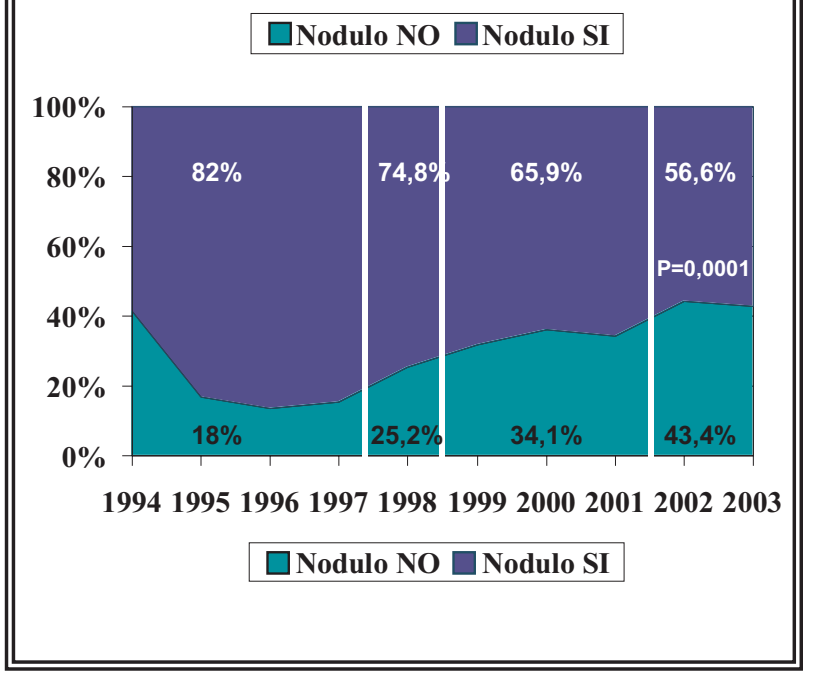

FIGURA 3. Evolución de la existencia de nódulo ecográfico en los pacientes sometidos a ETR-biopsia (gráfico superior) y en los pacientes con diagnóstico de cáncer de próstata (gráfico inferior). 


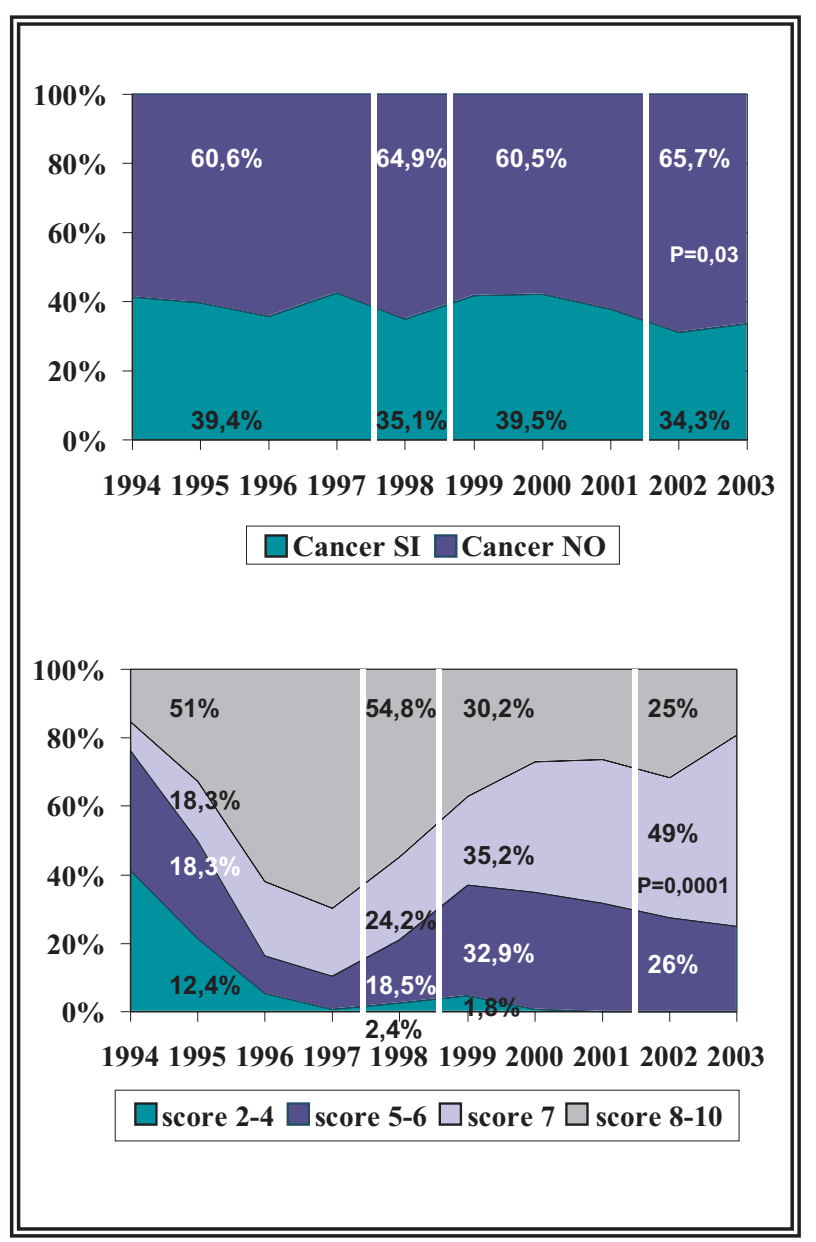

FIGURA 4. Evolución de la existencia de cáncer en la biopsia (gráfico superior) y del score de Gleason (gráfico inferior) en los pacientes con diagnóstico de cáncer de próstata.

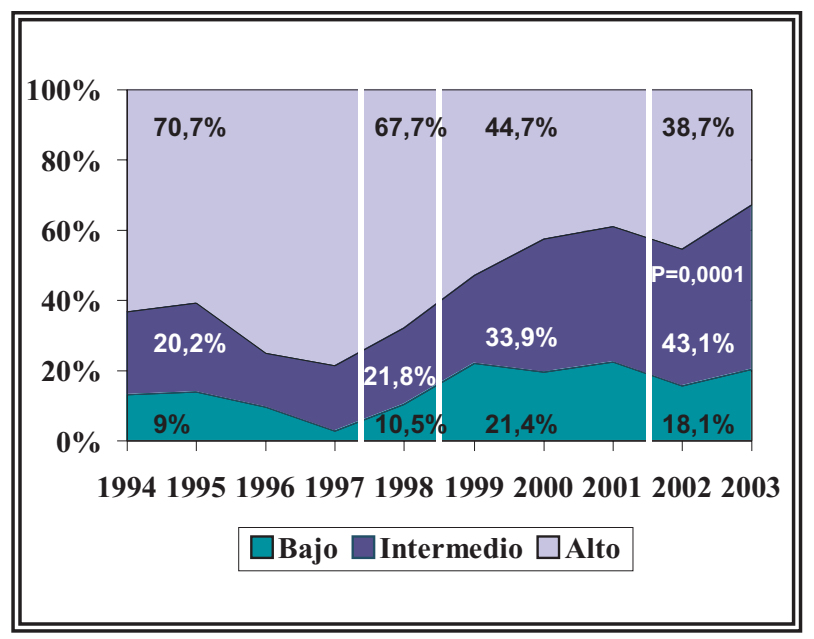

FIGURA 5. Evolución según su clasificación en grupos de riesgo en los pacientes con diagnóstico de cáncer de próstata.

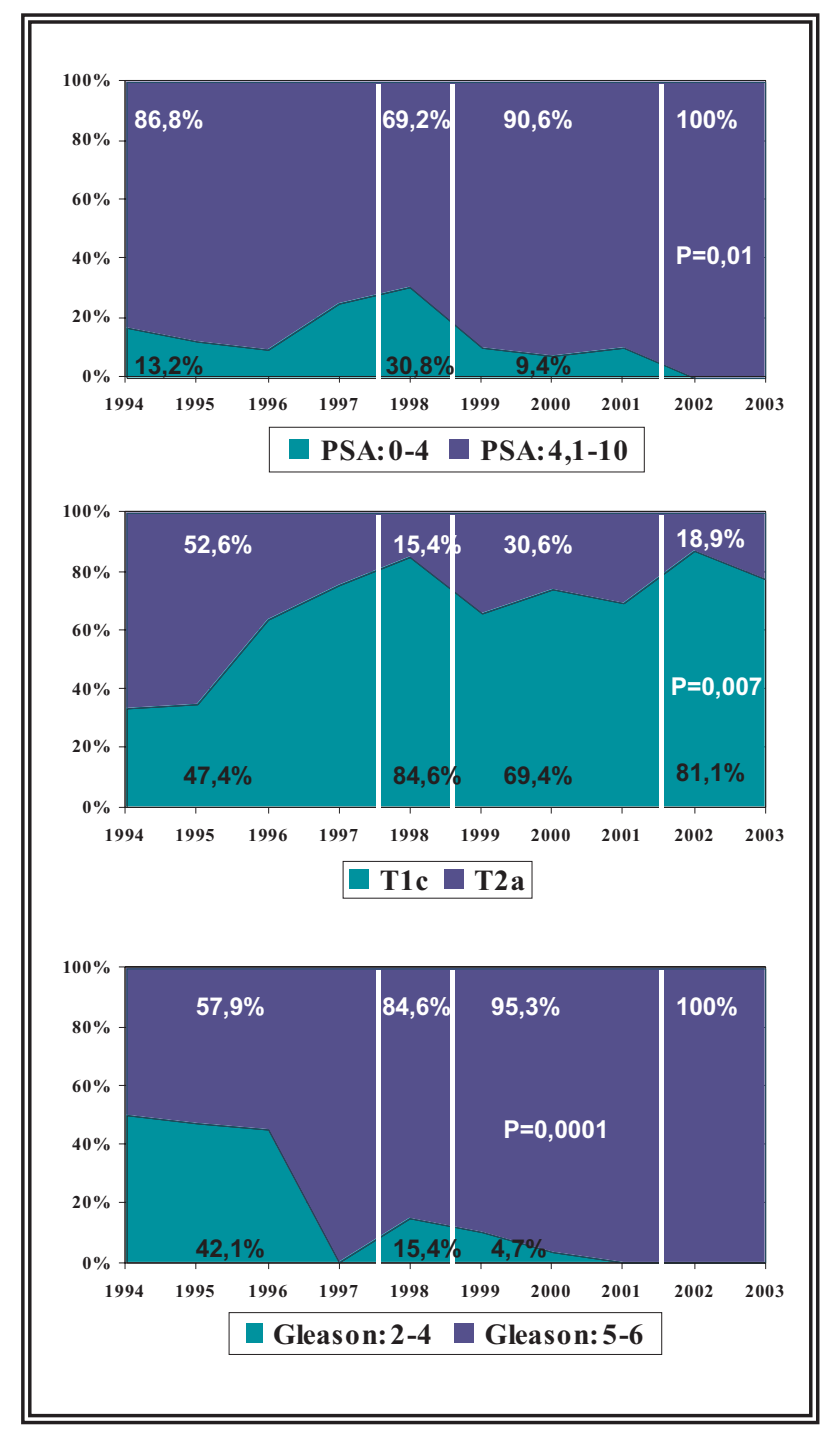

FIGURA 6. Evolución de los niveles del PSA, hallazgos al tacto rectal y score de Gleason en los pacientes con cáncer de próstata y bajo riesgo.

\section{DISCUSIŌN}

El interés creciente por el diagnostico precoz del CP (incremento del uso del PSA como marcador tumoral en Atención Primaria, revisiones de empresa y consultas de Urologia) ha producido en estos últimos 10 años una serie de modificaciones, tanto en las características de los sujetos que son seleccionados para biopsia de próstata, como en los pacientes diagnosticados de CP.

En los pacientes remitidos para ETR y biopsia hemos observado, al igual que otros autores ${ }^{13}$, una disminución de la edad media, niveles más bajos de PSA, disminución de las anormalidades al tacto rectal y disminución de la existencia de 
alteraciones ecográficas en la próstata. La primera consecuencia de biopsiar a pacientes cuyos factores de riesgo de padecer un CP tienen menos peso, ha sido la disminución de la incidencia de biopsias positivas como se refleja también en las series de otros autores ${ }^{5}$. Este aumento en los falsos positivos supone que cada vez sometemos a más pacientes sin $\mathrm{CP}$ a las posibles complicaciones de la biopsia de próstata $^{14,15}$ y al estrés psicológico, a él y a sus familiares, que conlleva todo el proceso de indicación, realización y espera del resultado de la biopsia. Un porcentaje no despreciable de estos pacientes deberán de someterse a más de una biopsia debido a la aparición de PIN de alto $\operatorname{grado}^{16}$, atipia glandular ${ }^{17}$ o persistencia en la elevación de $\mathrm{PSA}^{18}$, esto supone un incremento en la utilización de los recursos y de los costes necesarios para el diagnostico de CP.

En los pacientes diagnosticados de CP también hemos observado una migración de los factores pronósticos clínicos: más estadios T1c, más pacientes con niveles de PSA entre 4 y 10 ng/ml. y menos pacientes con alteraciones ecográficas identificables, como otros autores ${ }^{13}$. Sin embargo hemos observado la desaparición de los score de Gleason 2-4, la disminución de los score 5-6 y 810, aumentando el score 7. La explicación a este aparente paradoja, mejora de los factores pronósticos clínicos y empeoramiento de los patológicos, se basa en los cambios de la interpretación del sistema de Gleason con el tiempo y a que su reproducibilidad tanto intra como interobservador se estima entre el $60 \%$ y el $90 \%{ }^{19}$. Smith y cols. ${ }^{20}$ revaluaron 23 biopsias de próstata realizadas entre 1989 y 1991 y 32 realizadas entre 1998 y 2000 para evaluar la variabilidad intraobservador (cada patólogo revisó las mismas biopsias que realizó inicialmente). Hubo un cambio significativo de score de Gleason en el 44\% de las biopsias del primer periodo y del 31\% en las del segundo.

En las biopsias de 1989-1991 después de la regradación se observó una supragraduación del $35 \%$ y una infragraduación del 9\%, mientras que en las biopsias de 1998-2000 los cambios fueron del 9\% y del 22\% respectivamente. Kondylis y cols. ${ }^{19}$ revaluaron 82 especimenes de biopsia de próstata realizadas entre 1975 y 1984 que inicialmente fueron clasificadas según el grado de diferenciación histológica como: bien (gleason 24) - 46\%, moderadamente (Gleason 5-7) - 34\% y pobremente diferenciado (Gleason 8-10) - 20\%. La clasificación tras la reevaluación fue: bien $1,2 \%$, moderadamente - 45\% y pobremente diferenciado - 52,6\%. La práctica desaparición en la reclasificación de los grados 2-4 es debido a que es preciso la existencia de un patrón de arquitectura nodular, lo cual es un hallazgo muy infrecuente en los especimenes de biopsia prostática. Este sesgo de interpretación lo atribuyen los autores a la experiencia acumulada por los patólogos y a la actualización cada vez con criterios más específicos de asignación del grado, pero no pueden excluir que se esté produciendo un cambio en la biología del tumor en el tiempo ${ }^{19,20}$.

Los cambios observados en las variables clínicas y patológicas conllevan a modificaciones a lo largo del tiempo en la asignación de los pacientes a los grupos de riesgo en función de las 3 variables valoradas (PSA, tacto y Gleason), sobre todo en el grupo de riesgo intermedio. Al comienzo del estudio más del 80\% de los pacientes que fueron asignados al grupo de riesgo intermedio lo fueron por presentar un PSA entre 10 y 20 ng/ml., mientras que al final más del 90\% de los pacientes lo fueron por un score de Gleason igual a 7 (Fig. 7).

A pesar del incremento en el Gleason por cambios en los criterios de asignación, debido a la disminución de los niveles del PSA y el aumento de los estadios T1c-2a es posible que el riesgo biológico de los pacientes del final del estudio sea mucho menor que los que tenían los sujetos al principio del estudio en este mismo grupo de riesgo. Estos hallazgos coinciden con los aportados por Cooperberg y cols. ${ }^{12}$ tanto en el grupo de riesgo intermedio como en el elevado.

En resumen, actualmente diagnosticamos a los pacientes de CP con unos factores pronósticos más favorables que hace una década. Pero el precio que tenemos que pagar por esta anticipación diagnóstica se traduce en: una menor efectividad de la biopsia, un aumento de la población sin CP sometida a las complicaciones físicas y al estrés psicológico que implica la realización de la biopsia, un aumento de pacientes sometidos a rebiopsia y por lo tanto, un incremento en la utilización de los recursos y de los costes necesarios para el diagnostico de CP. 


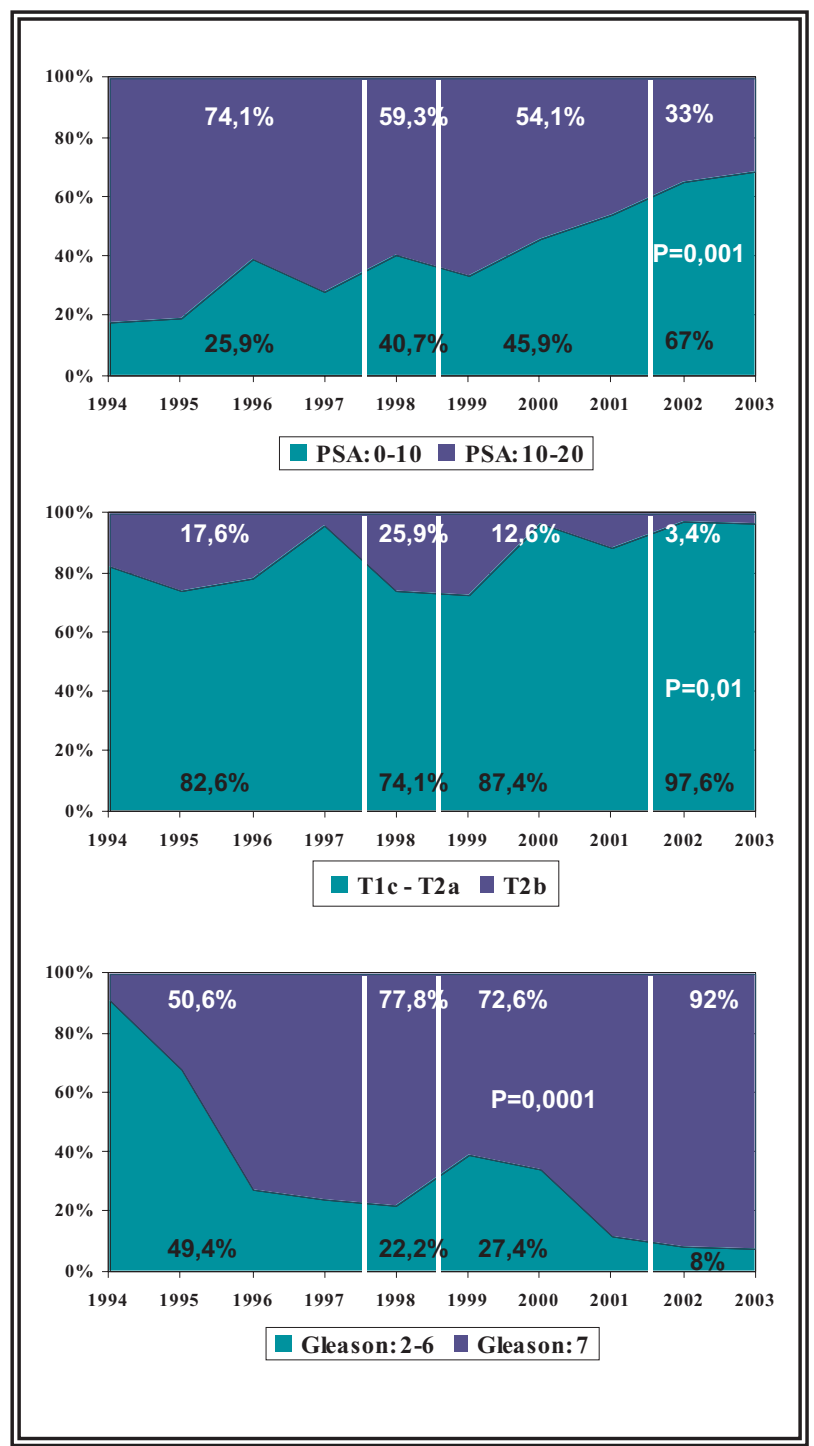

FIGURA 7. Evolución de los niveles del PSA, hallazgos al tacto rectal y score de Gleason en los pacientes con cáncer de próstata y risgo intermedio.

\section{REFERENCIAS}

1. Jemal,A; Tiwari,RC; Murray,T; y cols: Cancer Statistics, 2004. CA Cancer J Clinis. 2004. 54:8-29.

2. Herranz Amo,F; Arias Funez,F; Arrizabalaga Moreno,M; y cols.: El cáncer de próstata en la Comunidad de Madrid en el año 2000. I - Incidencia. Actas Urol Esp. 2003. 27:323334 .

3. Smith,RA; von Eschenbach,AC; Wender,R; et al.: American Cancer Society guidelines for the early detection of cancer: update of early detection guidelines for prostate, colorectal, and endometrial cancers AND update 2001: testing for early lung cancer detection. CA Cancer J Clin. 2001. 51:38-75.

4. Thompson,I; Carrol,P; Coley,C; y cols.: Prostate-specific antigen (PSA) best practice policy. Oncology. 2000. 14:267286.

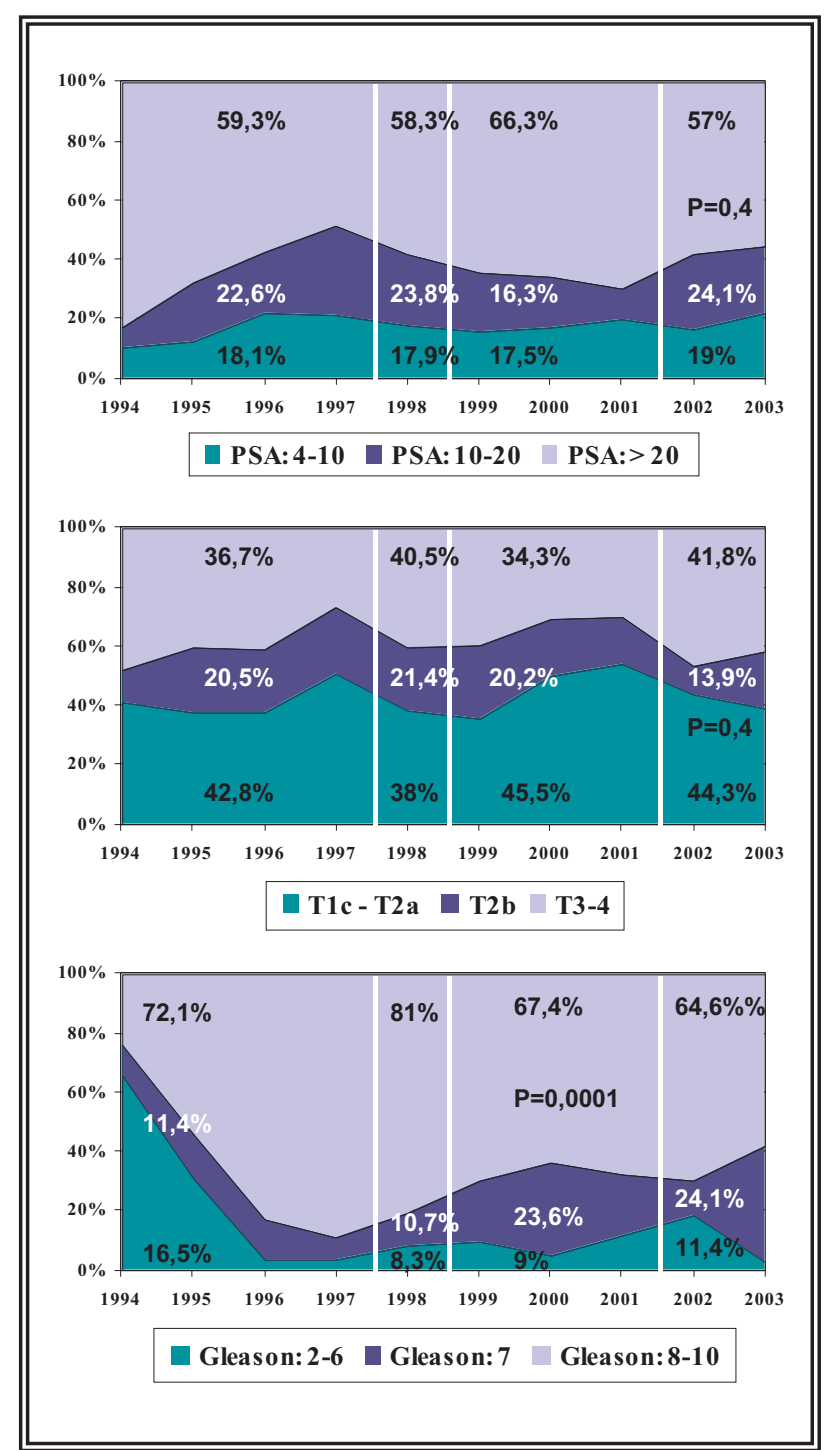

FIGURA 8. Evolución de los niveles del PSA, hallazgos al tacto rectal y score de Gleason en los pacientes con cáncer de próstata y riesgo elevado.

5. Roberts,RO; Bergstralh,EJ; Peterson,NR; y cols: Positive and negative biopsies in the pre-PSA and PSA era, 1980-1997. J Urol 2000. 163: 1471-1475.

6. Carvalhal,GF, Smith,DS; Mager,DE; y cols: Digital rectal examination for detecting prostate cancer at prostate specific antigen levels of $4 \mathrm{ng} . / \mathrm{ml}$. or less. J Urol 1999. 161: 835-839.

7. Ravery,V; Billebaud,T; Toublanc,M, y cols: Diagnostic value of ten systematic TRUS-guided prostate biopsies. Eur Urol 1999. 35: 298-303.

8. Jacobsen,SJ; Katusic,SK; Bergstralh,EJ; y cols: Incidence of prostate cancer diagnosis in the eras before and after serum prostate-specific antigen testing. JAMA. 1995. 274: 1445-1449.

9. Jania,AB; Vaida,F; Hanks, G; y cols: Changing face and fifferent countenances of prostate cancer: racial and geograp 
hics differences in prostate-specific antigen (PSA), stage, and grade trands in the PSA era. Int $J$ Cancer. 2001. 96: 363-371.

10. Herranz Amo,F; Verdú Tartajo,F; Díez Cordero,JM; y cols.: Utilidad del cociente PSA libre / PSA total en el diagnóstico del cáncer de próstata en pacientes sintomáticos con PSA entre 2,5 y $20 \mathrm{ng} / \mathrm{ml}$. Actas Urol.Esp. 2000. 24:24-30.

11. D'Amico, AV; Whittington,R; Malkowicz,SB; Schultz,D; Blank,K; Broderick, GA; y cols.: Biochemical outcome after radical prostatectomy, external beam radiation therapy, or interstitial radiation therapy for clinically localized prostate cancer. JAMA, 1998. 280: 969-974.

12. Cooperberg,MR; Lubeck,DP; Mehta,SS; Carroll, PR: Time trends in clinical risk stratification for prostate cancer: implications for outcomes (data from CaPSURE). J.Urol 2003.170:S21-S27.

13. Roberts,RO; Bergstralh,EJ; Lieber,MM; Jacobsen,SJ: Digital rectal examination and prostate-specific antigen abnormalities at the time of prostate biopsy and biopsy outcomes, 1980 to 1997. Urology. 2000. 56:817-822.

14. Herranz Amo,F; Rodríguez Fernández,E; Díez Cordero, $\mathrm{JM}^{\mathrm{a}}$; y cols: Morbilidad y tolerancia de la biopsia transrectal ecodirigida de la próstata. Actas Urol Esp. 1996. 20:858-866.

15. Rietbenger,JB; Kruger,AE; Kranse,R; y cols: Complications of transrectal ultrasound-guided systematic sextant biopsies of the prostate: evaluation of complications rates and risk factors within a population-based screening program. Urology. 1997. 49:875-880.
16. Herranz Amo,F; Álvarez Fernández,E; Díez Cordero,JMª .; y cols: Incidencia de la neoplasia intraepitelial prostática de alto grado en las biopsias transrectales de próstata. Arch Esp Urol. 2001. 54:321-326.

17. Herranz Amo,F; Verdú Tartajo,F; Díez Cordero,JMá; y cols: Incidencia y significación de la "proliferación acinar atípica" en las biopsias transrectales de próstata. Actas Urol Esp. En prensa.

18. Herranz Amo,F; Díez Cordero,JMá; Verdú Tartajo,F; y cols: Rentabilidad diagnóstica de la biopsia transrectal ecodirigida repetida en función de los hallazgos clínicos y anatomopatológicos. Arch Esp Urol. 1999. 23:394-399.

19. Kondyls,FI; Moriarty,RP; Bostwick,D; Schellhammer,PF: Prostate grade assignment: the effect of chronological, interpretative and translation bias. J.Urol. 2003. 170: 1189-1193.

20. Smith,EB; Frierson,HF; Mills,SE; Boyd,JC; Theodorescu, D: Gleson scores of prostate biopsy and radical prostatectomy specimens over the past 10 years. Cancer. 2002. 94:2282-2287.

\author{
Dr. F. Herranz Amo \\ C/Doctor Esquerdo 155 $5^{\mathrm{a}}, 7^{\circ}, 3$ \\ 28007 - Madrid
}

(Trabajo recibido el 15 mayo de 2004) 